\title{
Collective Force Generation by Molecular Motors Is Determined by Strain-Induced Unbinding
}

\author{
Mehmet Can Uçar*, ${ }^{*} \ddagger$ and Reinhard Lipowsky ${ }^{\ddagger}$ (i) \\ ${ }^{\dagger}$ Institute of Science and Technology Austria, Am Campus 1, 3400 Klosterneuburg, Austria \\ ${ }^{\ddagger}$ Max Planck Institute of Colloids and Interfaces, Am Mühlenberg 1, 14476 Potsdam, Germany
}

Supporting Information

ABSTRACT: In the living cell, we encounter a large variety of motile processes such as organelle transport and cytoskeleton remodeling. These processes are driven by motor proteins that generate force by transducing chemical free energy into mechanical work. In many cases, the molecular motors work in teams to collectively generate larger forces. Recent optical trapping experiments on small teams of cytoskeletal motors indicated that the collectively generated force increases with the size of the motor team but that this increase depends on the motor type and on whether the motors are studied in vitro or in vivo. Here, we use the theory of stochastic processes to describe the motion of $\mathrm{N}$ motors in a stationary optical trap and to compute the $\mathrm{N}$ dependence of the collectively generated forces. We consider six distinct motor types, two kinesins, two dyneins, and two myosins. We show that the force increases always linearly with $N$ but with a prefactor that depends on the performance of the single motor. Surprisingly, this prefactor increases for weaker motors with a lower stall force. This counter-intuitive behavior reflects the increased probability with which stronger motors detach from the filament during strain generation. Our theoretical results are in quantitative agreement with experimental data on small teams of kinesin-1 motors.

KEYWORDS: Motor proteins, optical tweezers, kinesin, nanomachines, cargo transport, cooperative behavior

T $\mathrm{n}$ biological systems, directed motion at the nanoscale is 1 primarily driven by motor proteins. These biomolecular machines transduce the chemical free energy released from nucleotide hydrolysis into mechanical work, which is expended in diverse processes ranging from intracellular cargo transport ${ }^{1}$ to microtubule alignment and spindle formation in mitosis. ${ }^{2,3}$ Superfamilies of kinesin, dynein, and myosin represent cytoskeletal motors that move on microtubules and actin filaments and typically cooperate in teams consisting of several motors. $^{4-6}$ An improved understanding of the behavior of motor teams is necessary to elucidate, e.g., cargo transport over distances ranging from micrometers to a meter such as in neurons ${ }^{7,8}$ and becomes increasingly relevant for the production of re-engineered or de novo motors ${ }^{9}$ for prospective nanotechnological applications, such as in sensitive biosensing and diagnostics beyond microfluidics. ${ }^{10}$

Properties of single molecules, such as the force generation or stepping dynamics of a single motor, can be systematically explored by in vitro optical trapping experiments. ${ }^{11}$ In addition to the single-molecule techniques, DNA-based motor assemblies make it possible to control the number of motors within a team and to characterize the collective dynamics, e.g., via run length and velocity measurements, as well as precise detection of intermotor distances. ${ }^{12,13}$ Furthermore, forces generated collectively by motor teams can be directly measured using stationary optical traps, both in vivo and in vitro. From such measurements, both "additive" and "subadditive" behavior has been proposed: For kinesin-1 in vitro, the collectively generated force was found to be much smaller than the sum of the single-motor forces, corresponding to "subadditive" behavior. ${ }^{14,15}$ On the other hand, the measurements on kinesin-1 in vivo, ${ }^{16}$ kinesin-5, ${ }^{3,17}$ and cytoplasmic dynein $^{18-20}$ indicated that, for these motors, the collectively generated force is almost equal to the sum of the single-motor forces.

In this paper, we develop a unifying theoretical description that explains the diverse collective behavior of the different motor types in terms of their single-molecule properties. We first investigate forces generated by a single motor in a stationary optical trap and show that the average force of a single motor can deviate strongly from its stall force at which the motor stops. We then study collective forces generated by teams of kinesin- 1 and of two types of dynein motors and find subadditive force generation for kinesin- 1 and yeast dynein as well as almost additive force generation for mammalian dynein. We show that subadditive force generation cannot be explained

Received: October 28, 2019

Revised: November 28, 2019

Published: December 4, 2019 
by the force sharing configurations of active motors during strain generation. We compare our theoretical results with two sets of experimental data on kinesin-1 and find very good quantitative agreement. We furthermore study force generation of three additional motor types, including two types of myosins, and find that collective forces increase linearly with the number of motors in a team for all motor types. Surprisingly, weak motors with a low stall force can generate forces that are closer to their collective stall force as opposed to strong motors. Using a coarse-grained model, we finally link collectively generated forces to properties of single motors and predict that cooperativity in force generation improves considerably for teams consisting of motors with a low stall force and/or detachment rate, i.e., for weak and/or processive motors, respectively. In contrast, strong and/or slow motors exhibit impaired cooperativity because they frequently unbind from the track before reaching their collective stall force.

Single-Motor Properties. We consider a stationary optical trapping assay with a team consisting of $N$ available motors pulling on a bead; see Figure 1. Each motor can step forward

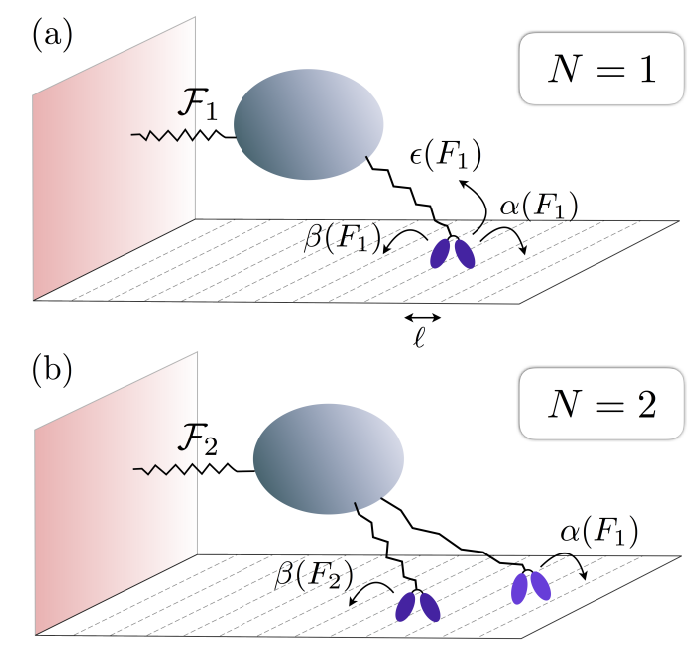

Figure 1. Force generation by molecular motors in a stationary optical trap. An arbitrary number $N$ of available motors, here illustrated for (a) $N=1$ and (b) $N=2$, can pull on a bead (gray sphere) and take discrete steps of size $l$ on a polarized track (here depicted by a $2 \mathrm{~d}$ surface). The spring attached to the wall on the left-hand side represents the laser field of the optical trap. Each motor with label $j$ stochastically steps forward and backward with force-dependent rates $\alpha\left(F_{j}\right)$ and $\beta\left(F_{j}\right)$, respectively, and unbinds from the track with the force-dependent unbinding rate $\epsilon\left(F_{j}\right)$. Detached (inactive) motors can rebind to the track with a constant rebinding rate $\pi_{0}$. The unbinding and rebinding events change the number $n$ of attached (active) motors with $0 \leq n \leq N$. Elastic forces $F_{j}$ corresponding to the extension of the motor linkers are in general different for each active motor, e.g., $F_{1} \neq F_{2}$ as depicted in panel $b$, and the optical trap measures the overall force $\mathcal{F}_{n}=\sum_{j}^{n} F_{j}$ at any instant.

and backward with force-dependent rates $\alpha(F)$ and $\beta(F)$, respectively. Motors can also unbind from their track with a force-dependent rate $\epsilon(F)$ and rebind to it with a constant rate $\pi_{0}$. As a consequence, at each time point, the number $n$ of active motors, i.e., motors that are attached to the track, can change stochastically with $0 \leq n \leq N$. The unbinding rate depends exponentially on the force acting on the motor, i.e., $\epsilon(F) \equiv \epsilon_{0} \exp \left(|F| / F_{\mathrm{d}}\right)$, where $\epsilon_{0}$ is the zero-force unbinding rate and $F_{\mathrm{d}}$ is the detachment force of a single motor. Forward and backward stepping rates are related to the force-velocity relation of a single motor via $\alpha(F)-\beta(F)=v(F) / l$, where $l$ is the step size of the motor. The velocity $v(F)$ is a monotonically decreasing function of the force acting on the motor with the zero-force velocity given by $v(F=0)=v_{0}$. When the force $F$ experienced by the motor reaches the stall force $F_{\mathrm{s}}$, its velocity becomes zero, i.e., $v\left(F=F_{\mathrm{s}}\right)=0$, and the motor steps forward and backward with equal probabilities due to $\alpha(F)=\beta(F)$. In general, one can define "strong" and "weak" motors using the ratio of stall force $F_{s}$ to detachment force $F_{\mathrm{d}}$. However, because the detachment force $F_{\mathrm{d}}$ varies only between 2 and $3 \mathrm{pN}$ for all motors studied here, whereas the stall force varies between 1 and $7 \mathrm{pN}$, we will consider motors with a stall force $F_{\mathrm{s}}<3 \mathrm{pN}$ as weak and with $F_{\mathrm{s}}>3 \mathrm{pN}$ as strong. For all parameter values used in this paper, which were obtained from refs 3, 17, and 20-37, see Table S1 in the Supporting Information. We note that, so far, a full set of motor parameters has only been measured for kinesin-1. For the other motors, we complemented the list of motor parameters by values that have been measured for different motor types. The choice of the motor parameters is explained in more detail in section S1.2 in the Supporting Information.

Forces Generated by a Single Motor. We first investigate the force generation by a single available motor $(N=1)$ in an optical trap, as illustrated in Figure 1a. Single steps taken by the motor as well as the detachment events lead to bead displacements that determine the instantaneous force $\mathcal{F}_{n}$ measured by the optical trap, where $n=1$ and $n=0$ correspond to active (attached) and inactive (detached) motor states, respectively. For $n=1$, the force measured by the optical trap is equal to the elastic force experienced by the motor, i.e., $\mathcal{F}_{1}=F_{1}$, whereas $\mathcal{F}_{0}=0$ for $n=0$, because the motor does not experience any forces when it is detached. The elastic force $F_{1}$ acting on the active motor is given by $F_{1}=\kappa_{\text {eff }} l s_{1}$ with the effective spring constant $\kappa_{\text {eff }} \equiv \kappa_{\mathrm{t}} \kappa_{\mathrm{m}} /\left(\kappa_{\mathrm{t}}+\right.$ $\left.\kappa_{\mathrm{m}}\right)$, where $\kappa_{\mathrm{t}}$ is the trap stiffness and $\kappa_{\mathrm{m}}$ is the spring constant of the motor linker. ${ }^{38}$ The step number $s_{1}$ corresponds to the distance of the motor from a relaxed configuration with $F_{1}=0$ in units of the step size $l$

We focus on force generation by kinesin- 1 and two different types of dyneins, strong and weak dynein, corresponding to yeast and mammalian cells, respectively. The difference between yeast and mammalian dynein is likely to arise from a C-terminal "cap" absent in yeast dynein, which regulates dynein's force output and processivity. ${ }^{39}$ Although dynein's force generation remains controversial with different in vivo and in vitro behavior, ${ }^{40}$ we use yeast and mammalian dynein to represent generic strong plus slow and weak plus fast types of motors, respectively; ${ }^{4}$ see Table $S 1$ in the Supporting Information for the corresponding parameter values.

Figure 2 displays exemplary trajectories of single motors pulling a bead in an optical trap as obtained from Monte Carlo simulations; see section S1.3 in the Supporting Information for details. The trajectories include regions where the single motor actively pulls on the bead, which leads to force generation, and regions with zero bead displacement following the singlemotor detachment events (orange arrows). The average singlemotor force $\left\langle\mathcal{F}_{1}\right\rangle$ is calculated by averaging over all force values generated during the active pulling of the single motor, i.e., excluding contributions from the detached motor states with $n$ $=0$. We observe that the average force $\left\langle\mathcal{F}_{1}\right\rangle$ can be (i) 
Single motor trajectories $(N=1)$
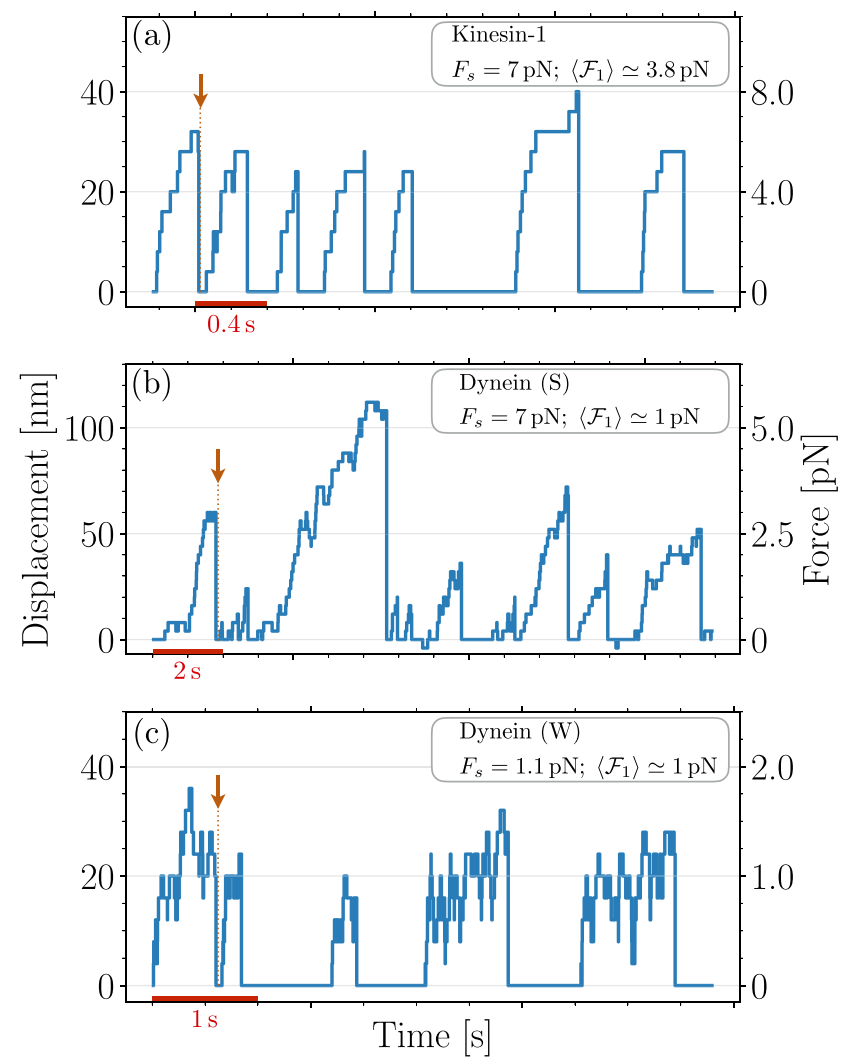

Figure 2. Segments of simulated trajectories of single motors pulling a bead in a stationary optical trap. Bead displacements and the instantaneous forces are plotted as a function of time for (a) kinesin-1, (b) strong (yeast) dynein, and (c) weak (mammalian) dynein. Scale bars for the time axis are shown in red. The arrows (orange) indicate exemplary detachment events of the active motors from the track. The average forces $\left\langle\mathcal{F}_{1}\right\rangle$ generated by the single motors and their stall forces $F_{s}$ are given in the insets. (a) For kinesin-1, the average force $\left\langle\mathcal{F}_{1}\right\rangle \simeq 3.8 \mathrm{pN}$ is somewhat lower than the stall force $F_{\mathrm{s}}=7 \mathrm{pN}$. (b) For strong dynein, the average force $\left\langle\mathcal{F}_{1}\right\rangle \simeq 1 \mathrm{pN}$ is very far from the stall force $F_{\mathrm{s}}=7 \mathrm{pN}$. (c) For weak dynein, the average force $\left\langle\mathcal{F}_{1}\right\rangle \simeq 1$ $\mathrm{pN}$ and the stall force $F_{\mathrm{s}}=1.1 \mathrm{pN}$ are very close.

somewhat lower than, (ii) very far from, or (iii) very close to the stall force $F_{s}$ of the single motor: Although kinesin-1 and strong dynein have the same stall force of $F_{\mathrm{s}}=7 \mathrm{pN}$, the average force is $\left\langle\mathcal{F}_{1}\right\rangle \simeq 3.8 \mathrm{pN}$ for kinesin-1 (see Figure 2a), which is somewhat lower than its stall force, and $\left\langle\mathcal{F}_{1}\right\rangle \simeq 1 \mathrm{pN}$ for strong dynein, which largely deviates from its stall force value (see Figure $2 \mathrm{~b}$ ). On the other hand, weak dynein's average force $\left\langle\mathcal{F}_{1}\right\rangle \simeq 1 \mathrm{pN}$ takes almost the same value as its stall force $F_{\mathrm{s}}=1.1 \mathrm{pN}$ (see Figure $2 \mathrm{c}$ ). We note that strong dynein builds up elastic forces very slowly (see the scale bar in Figure $2 \mathrm{~b}$ ) and often unbinds from the track at force values much smaller than its stall force.

Collective Force Generation by $\boldsymbol{N}$ Motors. We now consider collective force generation by teams consisting of an arbitrary number $N$ of available motors. The sum of forces experienced by the active motors of the team is equal to the overall force acting on the optical trap, i.e.,

$$
\mathcal{F}_{n} \equiv \sum_{j=1}^{n} F_{j}
$$

where $F_{j}$ is the elastic force acting on the $j$-th active motor, determined by the extension of the motor linker. Recall that, at any instant, the number of active motors fulfils $0 \leq n \leq N$. We furthermore define the elastic displacements $u_{j} \equiv L_{j}-L_{\|}$, where $L_{j}$ is the linker length with the rest length given by $L_{\|}$. As a reference configuration, we define a relaxed state of the system by $\mathbf{u}=\mathbf{u}_{0} \equiv\left(u_{1}=0, \ldots, u_{n}=0\right)$, where linkers of all active motors are relaxed, and assume that active motors can attain this relaxed state by taking discrete steps on the track. The forces $F_{j}$ acting on individual motors are then determined by the force balance with the optical trap as ${ }^{42}$

$$
F_{j} \equiv \kappa_{\mathrm{m}} u_{j}=\kappa_{\mathrm{m}} l\left[\left(1-\kappa_{\mathrm{m}} \varphi\right) s_{j}-\kappa_{\mathrm{m}} \varphi \sum_{\substack{k=1 \\ k \neq j}}^{n} s_{k}\right]
$$

where $\varphi \equiv 1 /\left(\kappa_{\mathrm{t}}+n \kappa_{\mathrm{m}}\right)$ is related to the shift in the bead position by each individual motor step; see section S1.1 in the Supporting Information for a derivation of eq 2 . As mentioned above, the step numbers $s_{j}$ correspond to the distances of the individual motors from the relaxed state $\mathbf{u}_{0}$ measured in units of the step size $l$. The overall instantaneous force $\mathcal{F}_{n}$ acting on the optical trap (see eq 1 ) is thus determined by the set of step numbers $\left\{s_{1}, s_{2}, \ldots, s_{n}\right\}$ in each activity state $(n)$ with $n$ active motors attached to the filament. The rebinding of an unbound motor to the filament is taken to lead to a bound motor with a relaxed or minimally stretched linker, depending on the preceding configuration of all bound motors. ${ }^{42}$ As a consequence, the overall force $\mathcal{F}_{n}$ acting on the trap remains constant or is minimally changed after each rebinding event. The average collective force $\left\langle\mathcal{F}_{N}\right\rangle$ for a team of $N$ available motors can then be calculated by weighting the instantaneous forces $\mathcal{F}_{n}$ by the dwell time of the system in the corresponding configuration, normalized by the total dwell time in activity states with $n=1, \ldots, N$; see section $S 1.3$ in the Supporting Information for details.

We furthermore introduce a coarse-grained model to estimate the average forces obtained from the "fine-grained" simulations: The coarse-graining algorithm is explicitly based on the assumption that the instantaneous force $\mathcal{F}_{n}$ acting on the optical trap is shared equally by all active motors. Furthermore, in the coarse-grained model, we ignore transitions between elastic substates of different activity states $(n)$ and subsequently reconnect these distinct activity states by effective rebinding and unbinding rates. These two assumptions considerably simplify the state space of the motor system, which allows a straightforward numerical calculation of coarsegrained average forces $\left\langle\mathcal{F}_{N}^{*}\right\rangle$. A detailed description of the coarse-grained model is provided in section S2 of the Supporting Information.

It is instructive to distinguish between two notions of additivity with respect to collective force generation by motor teams: First, one can choose the average force $\left\langle\mathcal{F}_{1}\right\rangle$ generated by a single available motor as the basic force scale and define additive force generation by the motor team to imply the collective force $\left\langle\mathcal{F}_{N}\right\rangle_{1} \equiv N\left\langle\mathcal{F}_{1}\right\rangle$. Alternatively, one may choose the stall force $F_{s}$ of a single motor as the basic force scale and take additive force generation to mean that the average force generated by the motor team is given by $\left\langle\mathcal{F}_{N}\right\rangle_{\mathrm{s}} \equiv N F_{\mathrm{s}}$. Recall that the average single-motor force is in general bounded by 

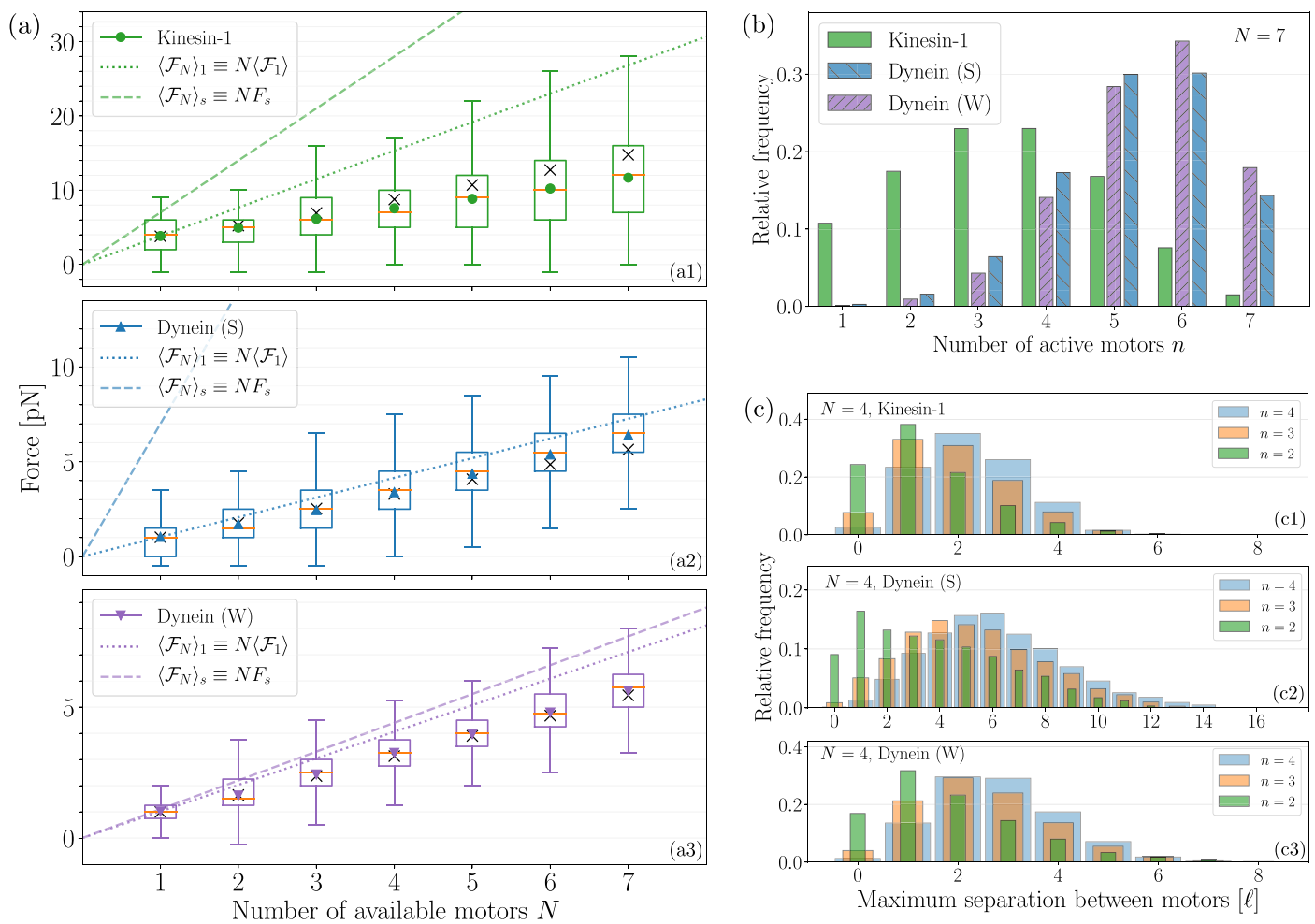

Figure 3. (a) Force generation by teams of kinesin and dynein motors consisting of up to $N=7$ motors in a stationary optical trap. Force distributions of (a1) kinesin-1, (a2) strong dynein (S), and (a3) weak dynein (W) are represented as box plots. In each box, mean values are given by triangular or circular markers and medians by horizontal lines (orange); whiskers indicate 1.5 interquartile range. The force scale $\left\langle\mathcal{F}_{N}\right\rangle_{1}$ and the collective stall force $\left\langle\mathcal{F}_{N}\right\rangle_{s}$ (see insets) are delineated by dotted and dashed lines in each plot, respectively. Average forces generated by kinesin-1 and strong dynein deviate strongly from their collective stall force values. In contrast, average forces of weak dynein are closer to its collective stall force values. The crosses represent average forces $\left\langle\mathcal{F}_{N}^{*}\right\rangle$ obtained from the coarse-grained model (see main text), which agree well with the finegrained simulation results for up to $N=5$ motors. (b) Relative frequencies of different activity states with $n$ active motors pulling on the bead for a total number of $N=7$ available motors. The probabilities for kinesin-1 decrease strongly for large $n$, in contrast with dynein motors, which have maximal probabilities around $n=6$. (c) Relative frequencies of the maximum separation between the leading and trailing motors for different numbers $n \leq N=4$ of active motors. The distances are given in units of the step size $l=8 \mathrm{~nm}$. For teams of kinesin-1 (c1) and of weak dynein (c3), the motors are typically separated by at most two and three steps, respectively, whereas, for teams of strong dynein (c2), the maximum motor-motor separations have a broader distribution, indicating a less uniform sharing of the overall load among the strong dyneins.

the stall force, i.e., $\left\langle\mathcal{F}_{1}\right\rangle \leq F_{s}$, and can take much smaller values than $F_{s}$ depending on the motor type, as shown in Figure $2 \mathrm{~b}$ for strong dynein. Therefore, the force scale $\left\langle\mathcal{F}_{N}\right\rangle_{1}$ will in general be smaller than the collective stall force $\left\langle\mathcal{F}_{N}\right\rangle_{s}$ and hence provides a weaker condition for the question of cooperativity. We will thus focus on the collective stall force as a measure of additive force generation of a motor team.

Collective Force Generation by Kinesin-1 and Dynein. Figure 3a displays the force distributions of kinesin1 and dynein teams for different numbers $N$ of available motors. We observe that average forces (triangular or circular markers) generated by kinesin-1 and strong dynein deviate strongly from their collective stall force values $\left\langle\mathcal{F}_{N}\right\rangle_{s}$ (dashed lines); see Figure 3a1,a2. In contrast, average forces of weak dynein are closer to its collective stall force $\left\langle\mathcal{F}_{N}\right\rangle_{s}$; see Figure 3a3. Interestingly, average forces of strong dynein are close to the force scale $\left\langle\mathcal{F}_{N}\right\rangle_{1}$ (dotted line) (see Figure 3a2); i.e., as a team, strong dynein can generate multiples of its single-motor average force $\left\langle\mathcal{F}_{1}\right\rangle \simeq 1 \mathrm{pN}$. Nevertheless, the average forces remain remarkably below the collective stall force because of strong dynein's poor performance as a single motor. Likewise, kinesin's force generation is also clearly subadditive, but the average force for a kinesin team of, e.g., $N=3$ available motors is close to the average force of a team of dyneins with $N=7$ motors. Estimates for the average forces $\left\langle\mathcal{F}_{N}^{*}\right\rangle$ obtained from the coarse-grained (CG) model are represented by crosses in each box plot and agree well with the fine-grained (FG) simulation results $\left\langle\mathcal{F}_{N}\right\rangle$ for up to $N=5$ available motors.

To obtain a mechanistic insight for the different $\mathrm{N}$ dependence of the collective forces by kinesin- 1 and dynein, we focus on probabilities of different activity states $(n)$ with 1 $\leq n \leq N$ active motors. These probabilities are estimated from the relative frequencies of the activity states $(n)$, determined by the normalized dwell times $\tau_{n} / T$, where $\tau_{n}$ represents the total time spent in activity state $(n)$ and the overall time is given by $T=\sum_{n=1}^{N} \tau_{n^{n}}$. Figure $3 \mathrm{~b}$ displays the relative frequencies of the different activity states $(n)$ for teams of kinesin-1, strong dynein, and weak dynein consisting of $N=7$ available motors. Both types of dyneins have higher probabilities for having a large number $n$ of active motors compared with kinesin- 1 . The increased number of active motors for dynein teams indicates that these teams can generate forces more persistently as opposed to kinesin-1. However, only weak dynein remains largely unaffected by strain-induced unbinding events both on the single-molecule level (see Figure 2c) and collectively (see Figure 3a3). 
One possible mechanism that might influence collective force generation concerns force sharing configurations of active motors during strain generation, as discussed in refs 20 and 42-45. To investigate how the overall load is shared by active motors during force generation, we display the relative frequencies of the maximum separation between the leading and trailing motors for teams consisting of $N=4$ available kinesins and dyneins in Figure 3c. These motor-motor separations are given in units of the step size $l=8 \mathrm{~nm}$. We observe that, in all activity states with $n$ active motors, kinesin1 and weak dynein motors are typically separated by at most two and three steps, respectively; see Figure $3 \mathrm{cl}$ and $\mathrm{c} 3$. In contrast, distributions for maximum motor separations for strong dynein span a wide range of values, which indicates that the overall load is not distributed as uniformly among team members as in the case of kinesin- 1 and weak dynein; see Figure $3 c 2$. Note that, in Figure $3 c$, the distributions for different $n$ are normalized and thus do not reflect the different frequencies of activity states $(n)$ shown in Figure $3 \mathrm{~b}$. In summary, these results demonstrate that subadditive force generation of kinesin-1 and strong dynein is related to the frequent unbinding events of the individual motors during strain generation and not to force sharing configurations of the active motors. In fact, when strain-induced unbinding events are largely suppressed, collective average forces do approach the collective stall force $\left\langle\mathcal{F}_{N}\right\rangle_{s}$, as we show for kinesin-1 in section S5 of the Supporting Information.

Comparison with Optical Trapping Experiments. Forces generated by a controlled number of kinesin-1 motors were experimentally studied in a stationary optical trapping assay in Furuta et al. ${ }^{15}$ using rat kinesin. In this study, individual kinesin motors were attached to a DNA scaffold with a separation of either 6 or $22 \mathrm{~nm}$ between two neighboring motors. In both cases, the number $N$ of available motors was systematically increased up to $N=4$, and it was found that average collective forces increased subadditively with $N$; see the experimental data in Figure 4. These average forces were obtained from histograms of maximal forces reached before detachment events of single motors. Using Monte Carlo simulations, we determined these force distributions by only changing the rebinding rate of kinesin-1 (see Table S1 in the Supporting Information), which is a forceindependent parameter that strongly depends on experimental buffer conditions. Our simulation results are also included in Figure 4 and show a very good agreement with the experimental ones. Mean and median values of the forces generated by $N \leq 4$ kinesins increase with $N$, but the collective force generation is clearly subadditive. Similar to Figure $3 b$, the probabilities to find $n$ active kinesin motors pulling the bead simultaneously against the trap decrease strongly as $n$ increases; see section S3 in the Supporting Information for details. This result again demonstrates that the collective force generation by multiple kinesins is strongly impeded by straininduced unbinding of the motors from the filament.

Collective Average Forces of Different Motor Types. To gain further insight into single-molecule properties that predominantly determine the different collective force generation mechanisms, we investigate three additional motor types: kinesin-5, myosin-5a, and myosin-6. Figure 5 displays the rescaled average force $\left\langle\mathcal{F}_{N}\right\rangle / F_{\mathrm{s}}$ for the six studied types of motors for increasing number $N$ of available motors up to $N=7$. For all studied motor types, we obtain the

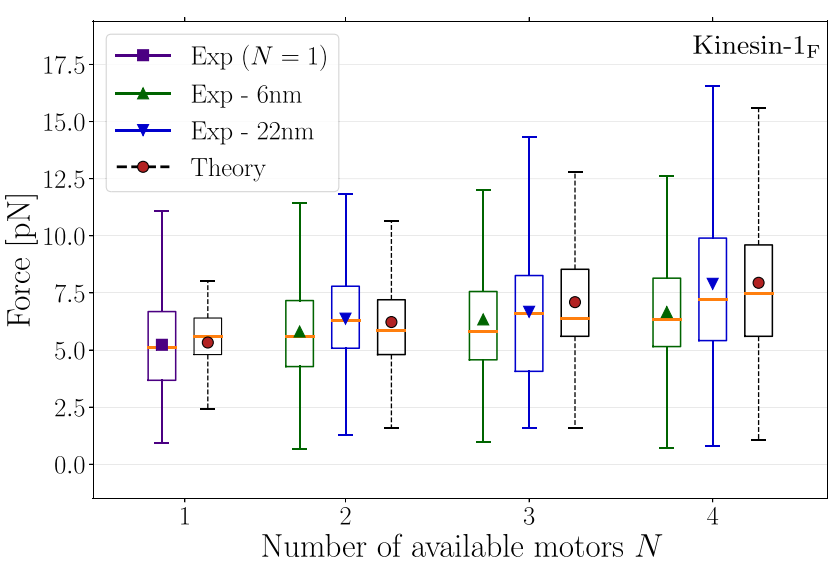

Figure 4. Comparison between simulation results and experimental data from Furuta et al. ${ }^{15}$ on force generation of kinesin- 1 for different numbers $N$ of available motors. In the latter study, two sets of experiments were performed with motors attached to a DNA scaffold with a separation of 6 or $22 \mathrm{~nm}$ between two neighboring motors. The distributions correspond to histograms of maximal forces generated during active pulling events before an attached motor detaches from the track. Box plots with dashed whisker lines represent the distributions obtained from the simulations. In each box, mean values are labeled by square, triangular, or circular markers and median values by the horizontal lines (orange). The theoretical results are in good agreement with the experimentally observed force values, which indicate a subadditive force generation mechanism for kinesin1.

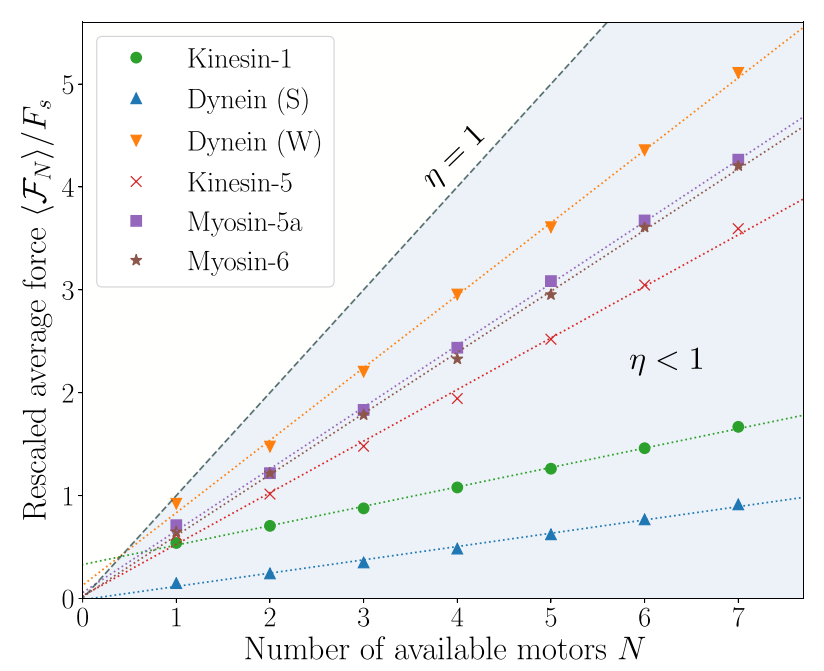

Figure 5. Collective force generation by different types of motors in a stationary optical trap for teams consisting of different numbers $N$ of available motors. Average collective forces $\left\langle\mathcal{F}_{N}\right\rangle$ are rescaled by the stall force $F_{\mathrm{s}}$ of a single motor. For all motor types, we observe a linear relationship between the rescaled average forces and the number of motors (dotted fit lines), i.e., $\left\langle\mathcal{F}_{N}\right\rangle / F_{s}=\eta N+c$ with a constant offset $c$ and a prefactor $\eta$ that take values between 0 and 1 . The shaded region represents $\eta<1$, whereas $\eta=1$ (dashed line) corresponds to average forces that are equal to the collective stall forces $\left\langle\mathcal{F}_{N}\right\rangle_{\mathrm{s}} \equiv N F_{\mathrm{s}}$. Kinesin-1 and strong dynein $(\mathrm{S})$ have a large stall force of $F_{s}=7 \mathrm{pN}$ and a prefactor of $\eta<0.2$, indicating a clearly subadditive force generation. In contrast, weak dynein (W), kinesin-5, myosin-5a, and myosin-6 have stall force values of $F_{\mathrm{s}} \leq 2.5 \mathrm{pN}$ and obtain a prefactor of $\eta>0.5$; i.e., they generate average forces that are closer to their collective stall forces. 
relationship $\left\langle\mathcal{F}_{N}\right\rangle / F_{s}=\eta N+c$; i.e., the forces increase linearly with the number $N$ of available motors. The values of the prefactor $\eta$ vary within the interval $0<\eta<1$. For the case $\eta=1$ and $c=0$, the average forces would be equal to the collective stall forces $\left\langle\mathcal{F}_{N}\right\rangle_{\mathrm{s}} \equiv N F_{\mathrm{s}}$. Furthermore, the offset $c$ vanishes for $\left\langle\mathcal{F}_{N}\right\rangle=N\left\langle\mathcal{F}_{1}\right\rangle$, i.e., for motors that can generate force additively with respect to the average single-motor force $\left\langle\mathcal{F}_{1}\right\rangle$. In fact, for the latter case, the prefactor $\eta$ is exactly given by the ratio $\left\langle\mathcal{F}_{1}\right\rangle / F_{\mathrm{s}}$ and is thus determined by the performance of a single motor. Apart from kinesin-1 and weak dynein, all studied motors are well described by $\left\langle\mathcal{F}_{N}\right\rangle \simeq N\left\langle\mathcal{F}_{1}\right\rangle$ (see Figure 3a and Figure $\mathrm{S} 4 \mathrm{a}$ ), which implies $\eta \simeq\left\langle\mathcal{F}_{1}\right\rangle / F_{\mathrm{s}}$. For all weak motors (dynein (W), kinesin-5, myosin-5a, and myosin6) with stall forces of $F_{\mathrm{s}} \leq 2.5 \mathrm{pN}$ and a ratio of $F_{\mathrm{s}} / F_{\mathrm{d}} \leq 1.2$, the prefactor satisfies $\eta>0.5$ as opposed to the two strong motors (kinesin-1 and dynein $(\mathrm{S}), F_{\mathrm{s}}=7 \mathrm{pN}$ ) with $F_{\mathrm{s}} / F_{\mathrm{d}} \geq 2.4$ for which the prefactor is $\eta<0.2$. The force distributions of kinesin-5, myosin-5a, and myosin- 6 from which the average forces are plotted here can be found in section S4 of the Supporting Information. We finally study a "test" motor by changing one parameter value at a time and find that, out of seven single-motor parameters, the collective force generation is most sensitive to changes in the parameters $F_{s}$ and $F_{\mathrm{d}}$, followed by those in the zero-force unbinding rate $\epsilon_{0}$ and the rebinding rate $\pi_{0}$. Moreover, our coarse-grained model predicts that the rescaled force $\left\langle\mathcal{F}_{N}^{*}\right\rangle / N F_{s}$ remains approximately constant if one changes $F_{\mathrm{s}}$ and $F_{\mathrm{d}}$ by the same factor, thereby keeping the force ratio $F_{\mathrm{s}} / F_{\mathrm{d}}$ fixed, as shown in section $\mathrm{S} 5$ of the Supporting Information.

Conditions of Approaching the Collective Stall Force. We now use our coarse-grained model to explore the parameter dependence of the coarse-grained average forces $\left\langle\mathcal{F}_{N}^{*}\right\rangle$. We set $N=3$ because the coarse-grained results agree well with the fine-grained simulation results for $N \leq 5$, as we show in Figure 3a1-a3 above. Figure 6 displays the rescaled average force $\left\langle\mathcal{F}_{N}^{*}\right\rangle / N F_{\mathrm{s}}$ as a function of the stall force $F_{\mathrm{s}}$, the zero-force unbinding rate $\epsilon_{0}$, and the zero-force velocity $v_{0}$. These three single-motor parameters modify the forcedependent rates and can be tuned experimentally; see the Discussion below. The remaining parameters in Figure 6a and $\mathrm{b}$ take the values corresponding to kinesin-1 and dynein, respectively; see Table S1 in the Supporting Information. We observe that the coarse-grained average forces $\left\langle\mathcal{F}_{N}^{*}\right\rangle$ approach the collective stall force $N F_{s}$ for motors with low stall force, low zero-force unbinding rate, and high zero-force velocity values. Variations in the stall force $F_{\mathrm{s}}$ and in the unbinding rate $\epsilon_{0}$ (see Figure 6a) have a stronger effect on the collective average forces compared with changes in the motor velocity $v_{0}$ (see Figure 6b). Rescaled collective forces $\left\langle\mathcal{F}_{N}^{*}\right\rangle / N F_{\text {s }}$ for kinesin-1 and dynein motors obtained from the coarse-grained model are very close to the fine-grained values of $\left\langle\mathcal{F}_{N}\right\rangle / N F_{s}$ for $N=3$, as listed in the caption of Figure 6. These results again demonstrate that weak motors can generate average forces that are closer to their collective stall forces, and suppressing detachment events leads to increased cooperativity in force generation.

Discussion. Using stochastic modeling, we investigated the mechanisms of collective force generation by arbitrary types of molecular motors and suggest a link between the performance of a single motor and the collective forces generated by small

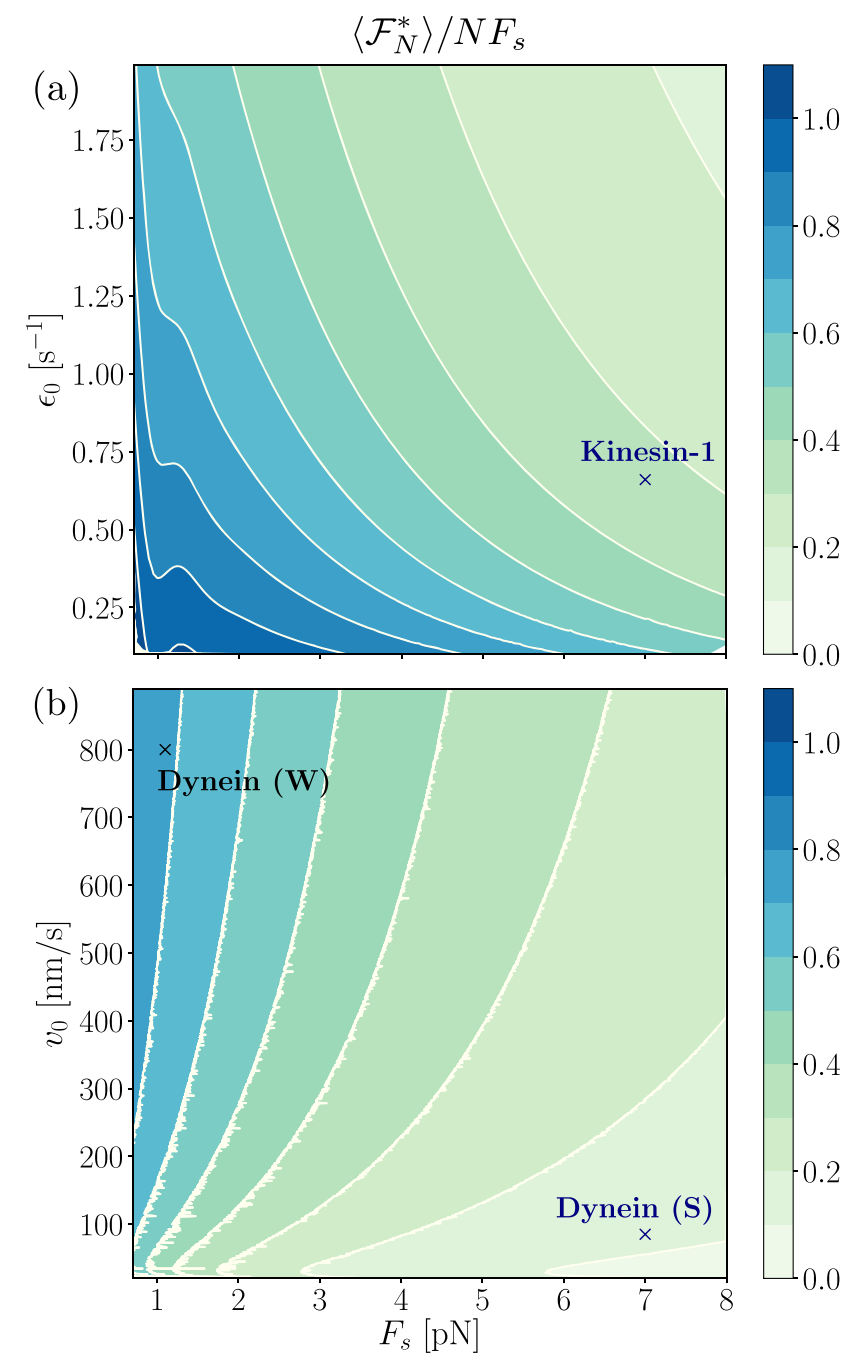

Figure 6. Parameter dependence of the collective average force $\left\langle\mathcal{F}_{N}^{*}\right\rangle$ for $N=3$ obtained from the coarse-grained model. The color bar shows the rescaled force $\left\langle\mathcal{F}_{N}^{*}\right\rangle / N F_{s}$. Low values of the stall force $F_{s}$ and of the zero-force unbinding rate $\epsilon_{0}$ (see panel a) as well as high values of the zero-force velocity $v_{0}$ (see panel $\mathrm{b}$ ) lead to increased cooperativity in terms of force generation. Remaining motor parameters take the values corresponding to (a) kinesin-1 and (b) dynein; see Table S1 in the Supporting Information. Marked values (crosses) for $\left\langle\mathcal{F}_{N}^{*}\right\rangle / N F_{s}$ with $N=3$ obtained from the coarse-grained (CG) model compared with the results from the fine-grained (FG) simulation are 0.329 (CG) vs 0.292 (FG) for kinesin-1, 0.12 (CG) vs 0.117 (FG) for strong dynein (S), and 0.718 (CG) vs 0.735 (FG) for weak dynein $(\mathrm{W})$.

teams of motors. In particular, we find that, in contrast with strong motors with a large stall force, weak motors cooperate better as a team in force generation (see Figure 5) and increasing the processivity or velocity of a single motor also leads to average forces that are closer to the collective stall force $N F_{\mathrm{s}}$ of a motor team; see Figure 6.

Existing hypotheses for the subadditive force generation of kinesin- 1 are based on unequal force sharing between motors and emphasize the velocity decrease of a single motor as the main underlying mechanism..$^{20,43-45}$ According to this argument, if the velocity of a single motor does not drop rapidly with increasing load, typical motor configurations will involve a single leading motor that carries the overall load. ${ }^{42}$ While the 
specific form of the force-velocity relation might affect the force sharing configurations of multiple motors, as studied for two motors in ref 43 , we claim that this mechanism is not sufficient to significantly modify the cooperativity in force generation; see section S6 in the Supporting Information for a brief discussion. Instead, we suggest that a simpler explanation for the subadditive force generation of kinesin- 1 is provided by the strain-induced unbinding events regardless of the force sharing configurations during strain generation, as indicated by Figure 3, as well as by the comparison with experimental data (see Figure 4 and section S3 in the Supporting Information).

In contrast with the subadditive force generation of kinesin-1 in vitro, evidence for additive force generation was found for both kinesin-1 and dynein teams in vivo. ${ }^{16}$ We note that, however, the stall force of kinesin-1 in vivo was substantially lower than its in vitro value $\left(\sim 2.5 \mathrm{pN}\right.$ in vivo ${ }^{16}$ vs $\sim 7 \mathrm{pN}$ in $v_{\text {vitro }}{ }^{25}$ ). Our model explicitly predicts increased cooperativity in force generation when the stall force is reduced (see Figure 6) and can thus explain this disparity between in vivo and in vitro observations. Nevertheless, stall force measurements in vivo can be difficult to interpret due to effects of molecular crowding such as the presence of accessory proteins ${ }^{16}$ or active opposing motors reducing the effective stall force of the motor team, ${ }^{36}$ and therefore, care must be taken before drawing a direct conclusion. In addition to the quantitative agreement between our theoretical results and experimental data on collective force generation by kinesin-1 (see Figure 4), our results of close to additive force generation for weak (mammalian) dynein and kinesin-5 (see Figure 5), are also in qualitative agreement with experimental observations of these motor types. ${ }^{3,17-20}$ Stochastic modeling has also been applied to teams of nonprocessive motors, such as myosin- $2 .{ }^{46,47}$ In the latter case, the collective forces saturated as a function of motor number, in contrast to the linear increase of the collective forces for processive motors as studied here; see Figure 5 .

Collective force generation of kinesin and dynein motors supports a rather balanced in vivo dynamics: although minusend directed dyneins cooperate better than plus-end directed kinesins as a team, the absolute force values of many dyneins are close to the forces of a few kinesins; see Figure 3a. Such a force-balanced tug-of-war with a large number of dyneins against a few kinesins is in accordance with several experimental observations. ${ }^{4,36,48}$ Moreover, dynein teams might exhibit persistent force generation because of the high number of active motors on average (see Figure $3 \mathrm{~b}$ ), in contrast with kinesin-1, which might generate forces more abruptly due to frequent unbinding events. A persistent force generation mechanism for dynein fits well with its role in continuous sliding of microtubules along the cortex during, e.g., spindle positioning. ${ }^{49}$

In principle, the three single-motor parameters $\epsilon_{0}, v_{0}$, and $F_{\mathrm{s}}$ that influence force generation by motor teams (see Figure 6) can be directly modified in experimental studies. Adding charged residues to the neck linker of kinesin-1, ${ }^{50}$ for example, strongly increases the processivity of a single motor, which determines the unbinding rate $\epsilon_{0}$. Furthermore, addition of LIS1 and NudE was found to increase both the processivity and force generation of dynein, ${ }^{34}$ and the removal of the Cterminal cap domain allows a switch from a weak to a strong motor. ${ }^{39}$ Finally, recent methods in engineering applications of molecular motors allowed the formation of three- and fourheaded myosins with velocities reaching up to $10 \mu \mathrm{m} / \mathrm{s}$ and average run lengths in $0.5-3 \mu \mathrm{m},{ }^{51}$ substantially increasing the native motor velocity and producing the fastest processive motor observed. Although these single-molecule modification techniques require further development, currently available experiments on collective movement and force generation by motor teams using DNA origami scaffolds allow a direct test for our predictions. ${ }^{13,15,52}$ Ideally, an iterative experimental methodology will enable a systematic analysis of the collective behavior of motor teams upon locally modifying features of single molecules. ${ }^{53}$

\section{ASSOCIATED CONTENT}

\section{Supporting Information}

The Supporting Information is available free of charge at https://pubs.acs.org/doi/10.1021/acs.nanolett.9b04445.

A detailed description of the two stochastic models, table of parameters, supplementary data for Figures 4 and 5, parameter dependence of the results, and an analysis on motors with different force-velocity functions (PDF)

Data obtained from the fine-grained simulations used in Figures 2-5, data obtained from the coarse-grained numerical calculations used in Figure 6, and a sample script for the fine-grained simulation as a Jupyter notebook (ZIP)

\section{AUTHOR INFORMATION}

\section{Corresponding Author}

*Email: mehmetcan.ucar@ist.ac.at.

ORCID $\odot$

Mehmet Can Uçar: 0000-0003-0506-4217

Reinhard Lipowsky: 0000-0001-8417-8567

Notes

The authors declare no competing financial interest.

\section{ACKNOWLEDGMENTS}

We thank Ken'ya Furuta (NICT) for sending us the optical trap data published in ref 15 and plotted in Figure ${ }^{4}$. M.C.U. thanks Levent Sağun (EPFL) and Katarzyna Ziółkowska (MPIKG) for their technical support as well as Jessica Huss (BOKU Vienna) and Tunç Yılmaz (U. Potsdam) for insightful comments.

\section{REFERENCES}

(1) Vale, R. D. Cell 2003, 112, 467.

(2) Cross, R. A.; McAinsh, A. Nat. Rev. Mol. Cell Biol. 2014, 15, 257.

(3) Shimamoto, Y.; Forth, S.; Kapoor, T. M. Dev. Cell 2015, 34, 669.

(4) Hendricks, A. G.; Holzbaur, E. L.; Goldman, Y. E. Proc. Natl. Acad. Sci. U. S. A. 2012, 109, 18447.

(5) Efremov, A. K.; Radhakrishnan, A.; Tsao, D. S.; Bookwalter, C. S.; Trybus, K. M.; Diehl, M. R. Proc. Natl. Acad. Sci. U. S. A. 2014, 111, No. E334.

(6) Nelson, S. R.; Trybus, K. M.; Warshaw, D. M. Proc. Natl. Acad. Sci. U. S. A. 2014, 111, No. E3986.

(7) Lipowsky, R.; Beeg, J.; Dimova, R.; Klumpp, S.; Müller, M. J. I. Phys. E 2010, 42, 649.

(8) Hirokawa, N.; Niwa, S.; Tanaka, Y. Neuron 2010, 68, 610.

(9) DelRosso, N. V.; Derr, N. D. Curr. Opin. Biotechnol. 2017, 46, 20.

(10) Korten, T.; Månsson, A.; Diez, S. Curr. Opin. Biotechnol. 2010, 21, 477.

(11) Veigel, C.; Schmidt, C. F. Nat. Rev. Mol. Cell Biol. 2011, 12, 163. 
(12) Lu, H.; Efremov, A. K.; Bookwalter, C. S.; Krementsova, E. B.; Driver, J. W.; Trybus, K. M.; Diehl, M. R. J. Biol. Chem. 2012, 287, 27753.

(13) Krementsova, E. B.; Furuta, K.; Oiwa, K.; Trybus, K. M.; Ali, M. Y. J. Biol. Chem. 2017, 292, 10998.

(14) Jamison, D. K.; Driver, J. W.; Rogers, A. R.; Constantinou, P. E.; Diehl, M. R. Biophys. J. 2010, 99, 2967.

(15) Furuta, K.; Furuta, A.; Toyoshima, Y. Y.; Amino, M.; Oiwa, K.; Kojima, H. Proc. Natl. Acad. Sci. U. S. A. 2013, 110, 501.

(16) Leidel, C.; Longoria, R. A.; Gutierrez, F. M.; Shubeita, G. T. Biophys. J. 2012, 103, 492.

(17) Fallesen, T.; Roostalu, J.; Duellberg, C.; Pruessner, G.; Surrey, T. Biophys. J. 2017, 113, 2055.

(18) Mallik, R.; Petrov, D.; Lex, S.; King, S.; Gross, S. Curr. Biol. 2005, 15, 2075.

(19) Soppina, V.; Rai, A. K.; Ramaiya, A. J.; Barak, P.; Mallik, R. Proc. Natl. Acad. Sci. U. S. A. 2009, 106, 19381.

(20) Rai, A. K.; Rai, A.; Ramaiya, A. J.; Jha, R.; Mallik, R. Cell 2013, $152,172$.

(21) King, S. J.; Schroer, T. A. Nat. Cell Biol. 2000, 2, 20.

(22) Altman, D.; Sweeney, H. L.; Spudich, J. A. Cell 2004, 116, 737.

(23) Leduc, C.; Campas, O.; Zeldovich, K. B.; Roux, A.; Jolimaitre, P.; Bourel-Bonnet, L.; Goud, B.; Joanny, J.-F.; Bassereau, P.; Prost, J. Proc. Natl. Acad. Sci. U. S. A. 2004, 101, 17096.

(24) Uemura, S.; Higuchi, H.; Olivares, A. O.; De La Cruz, E. M.; Ishiwata, S. Nat. Struct. Mol. Biol. 2004, 11, 877.

(25) Carter, N. J.; Cross, R. Nature 2005, 435, 308.

(26) Oiwa, K.; Sakakibara, H. Curr. Opin. Cell Biol. 2005, 17, 98.

(27) Toba, S.; Watanabe, T. M.; Yamaguchi-Okimoto, L.; Toyoshima, Y. Y.; Higuchi, H. Proc. Natl. Acad. Sci. U. S. A. 2006, 103, 5741.

(28) Gebhardt, J. C. M.; Clemen, A. E.-M.; Jaud, J.; Rief, M. Proc. Natl. Acad. Sci. U. S. A. 2006, 103, 8680.

(29) Reck-Peterson, S. L.; Yildiz, A.; Carter, A. P.; Gennerich, A.; Zhang, N.; Vale, R. D. Cell 2006, 126, 335.

(30) Valentine, M. T.; Fordyce, P. M.; Krzysiak, T. C.; Gilbert, S. P.; Block, S. M. Nat. Cell Biol. 2006, 8, 470.

(31) Gennerich, A.; Carter, A. P.; Reck-Peterson, S. L.; Vale, R. D. Cell 2007, 131, 952.

(32) Rogers, A. R.; Driver, J. W.; Constantinou, P. E.; Jamison, D. K.; Diehl, M. R. Phys. Chem. Chem. Phys. 2009, 11, 4882.

(33) Watanabe, T. M.; Iwane, A. H.; Tanaka, H.; Ikebe, M.; Yanagida, T. PLoS One 2010, 5, No. e12224.

(34) McKenney, R. J.; Vershinin, M.; Kunwar, A.; Vallee, R. B.; Gross, S. P. Cell 2010, 141, 304.

(35) Ali, M. Y.; Kennedy, G. G.; Safer, D.; Trybus, K. M.; Sweeney, H. L.; Warshaw, D. M. Proc. Natl. Acad. Sci. U. S. A. 2011, 108, No. E535.

(36) Blehm, B. H.; Schroer, T. A.; Trybus, K. M.; Chemla, Y. R.; Selvin, P. R. Proc. Natl. Acad. Sci. U. S. A. 2013, 110, 3381.

(37) Andreasson, J. O.; Milic, B.; Chen, G.-Y.; Guydosh, N. R.; Hancock, W. O.; Block, S. M. eLife 2015, 4, e07403.

(38) Berger, F.; Klumpp, S.; Lipowsky, R. Nano Lett. 2019, 19, 2598.

(39) Nicholas, M. P.; Höök, P.; Brenner, S.; Wynne, C. L.; Vallee, R. B.; Gennerich, A. Nat. Commun. 2015, 6, 6206.

(40) Torisawa, T.; Ichikawa, M.; Furuta, A.; Saito, K.; Oiwa, K.; Kojima, H.; Toyoshima, Y. Y.; Furuta, K. Nat. Cell Biol. 2014, 16, 1118.

(41) Blehm, B. H.; Selvin, P. R. Chem. Rev. 2014, 114, 3335.

(42) Uçar, M. C.; Lipowsky, R. Sci. Rep. 2019, 9, 454.

(43) Driver, J. W.; Jamison, D. K.; Uppulury, K.; Rogers, A. R.; Kolomeisky, A. B.; Diehl, M. R. Biophys. J. 2011, 101, 386.

(44) McLaughlin, R. T.; Diehl, M. R.; Kolomeisky, A. B. Soft Matter 2016, 12, 14.

(45) Wang, Q.; Diehl, M. R.; Jana, B.; Cheung, M. S.; Kolomeisky, A. B.; Onuchic, J. N. Proc. Natl. Acad. Sci. U. S. A. 2017, 114, No. E8611.

(46) Walcott, S.; Warshaw, D. M.; Debold, E. P. Biophys. J. 2012, 103, 501.
(47) Erdmann, T.; Albert, P. J.; Schwarz, U. S. J. Chem. Phys. 2013, $139,175104$.

(48) Sanghavi, P.; D'Souza, A.; Rai, A.; Rai, A.; Padinhatheeri, R.; Mallik, R. Curr. Biol. 2018, 28, 1460.

(49) Akhmanova, A.; Steinmetz, M. O. Nat. Rev. Mol. Cell Biol. 2015, 16,711 .

(50) Thorn, K. S.; Ubersax, J. A.; Vale, R. D. J. Cell Biol. 2000, 151, 1093.

(51) Schindler, T. D.; Chen, L.; Lebel, P.; Nakamura, M.; Bryant, Z. Nat. Nanotechnol. 2014, 9, 33.

(52) Derr, N. D.; Goodman, B. S.; Jungmann, R.; Leschziner, A. E.; Shih, W. M.; Reck-Peterson, S. L. Science 2012, 338, 662.

(53) Furuta, K.; Furuta, A. Curr. Opin. Biotechnol. 2018, 51, 39. 